\title{
A Rare Case of 83-Year-Old Transgender Female: Can Thyroid Hormone Deficiency Be Involved in Transgenderism and Gender Dysphoria?
}

\author{
Andrey Frolov, Lauren Polcaro, Craig Lawson, Yun Tan, John R. Martin III* \\ Center for Anatomical Science and Education, Department of Surgery, School of Medicine, Saint Louis University, \\ Saint Louis, Missouri, USA \\ Email: ^john.martin@health.slu.edu
}

How to cite this paper: Frolov, A., Polcaro, L., Lawson, C., Tan, Y. and Martin III, J.R. (2020) A Rare Case of 83-Year-Old Transgender Female: Can Thyroid Hormone Deficiency Be Involved in Transgenderism and Gender Dysphoria? Advances in Sexual Medicine, 10, 23-40.

https://doi.org/10.4236/asm.2020.102002

Received: February 12, 2020

Accepted: March 21, 2020

Published: March 24, 2020

Copyright $\odot 2020$ by author(s) and Scientific Research Publishing Inc. This work is licensed under the Creative Commons Attribution International License (CC BY 4.0).

http://creativecommons.org/licenses/by/4.0/

(c) (i) Open Access

\begin{abstract}
In the current report, we describe an 83-year-old biological male who selfidentified as a female by legally changing his first and middle names to female ones and whose death certificate states his sex as a female. The medical history of this individual indicated complete penectomy without further specification. Postmortem physical examination revealed an absence of penis with a large scrotum, transposed urethral orifice, and small testes. The histological analysis of the testes identified abnormal epithelium in the seminiferous tubules that lacked germ and Sertoli cells as well as the interstitium without Leydig cells present. The exome sequencing of the individual's DNA using the Next Generation Sequencing (NGS) Illumina platform revealed no genetic variants associated with either penile or urethral cancer that could have explained the complete penectomy, but pointed toward a potentially impaired production of T3 and T4 thyroid hormones which could account for the observed testicular malformation. Overall, the data obtained raise an important question as to whether the thyroid hormone axis could be an important part of the hormonal architecture supporting male sexual behavior.
\end{abstract}

\section{Keywords}

Transgenderism, Gender Dysphoria, Next Generation Sequencing, Thyroid Peroxidase, Cilia

\section{Introduction}

Transgender (TG) is a broad term, which describes an individual whose gender 
self-identification is different from the assigned sex at birth. When the latter becomes overwhelming and causes a significant psychological impact, the term gender dysphoria (GD) is used thereby presenting itself as an extreme case of TGism. The individuals with GD often seek a medical intervention, surgical or therapeutic, to correct the existing discordance between their gender self-identification and physical appearance [1].

As of 2016, approximately 1.4 million adults identify as TG in the United States [2]. The TG biological underlining is not well understood. Genetically, it appears that interplay between sex steroid receptor polymorphisms, particularly those of androgen and estrogen, which are associated with TG [3]. Yet the $R Y R 3$ variant was identified in the Han Chinese population with GD [4] and CYP17 single nucleotide polymorphism was linked to female-to-male TGism [5] whereas a common $S R D 5 A 2$ polymorphism has been shown recently to be benign in TGism (ClinicalTrials.gov Identifier: NCT00435513). Chromosomal aberrations, such as in Klinefelter syndrome (47, XXY), could also be responsible for GD in some individuals since a $1.13 \%$ frequency of patients with Klinefelter syndrome was found in GD population [6].

Metabolically, GD has been linked to androgen insensitivity in biological males. Individuals with complete androgen insensitivity syndrome (CAIS) have a 46, XY karyotype, bilateral testes, and female external genitalia [7]. These individuals tend to be psychosocially female and oriented toward men [8]. Individuals with partial androgen insensitivity syndrome (PAIS) also have a 46, XY karyotype, but may have penoscrotal hypospadias, micropenis, bifid scrotum, and undescended testes [9].

Most recently, a large cohort association study involving 380 transgender females (TFs) and 344 control male subjects revealed an over-representation in TFs of several allele combinations including androgen receptor (AR) that were proposed to feminize male individuals thereby leading to TGism [10].

Therefore, the main objective of this study was to provide in-depth examination of the current TF body to gain additional important insights into the respective biological underlinings through a systematic approach including gross anatomical dissection, histological analysis, and genetic screen using NGS technology.

\section{Methods}

\subsection{Human Cadaveric Body Procurement}

An 83-year-old TF cadaver was received through Saint Louis University (SLU) School of Medicine Gift of Body Program from an individual who had given his written informed consent. The body was embalmed through the right femoral artery with a mixture of water and a solution (2:1) containing $33.3 \%$ glycerin, $28.8 \%$ phenol, $4.6 \%$ formaldehyde, and 33.3\% methanol.

\subsection{Anatomical Dissection}

The cadaveric body was dissected according to [11]. 


\subsection{Histological Analysis}

Testicular tissue was procured from the embalmed body. Tissue fixation, paraffin embedding, sectioning, and staining with hematoxylin \& eosin were performed by Research Microscopy and Histology Core, Department of Pathology, Saint Louis University (SLU) School of Medicine according to the standardized procedures. Images were captured on Olympus 41BX-EPI microscope equipped with

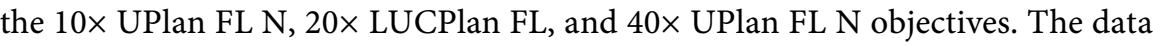
acquisition and image analysis were performed by using CellSens Standard software.

\subsection{Bone Densitometry}

Bone density of the lumbar spine and left femoral neck of the embalmed body was measured in triplicates using certified Hologic QDR-4500 X-ray Bone Densitometer in the dual energy X-ray absorptiometry (DEXA) mode following the manufacturer's protocol. The respective average T-score values were used throughout the text.

\subsection{Genetic Analysis}

The Next Generation Sequencing (NGS) and bioinformatics analysis were performed as previously described [12] [13] with the following modifications. DNA extracted from the tibia specimen procured from the embalmed TF body was sequenced to $30 \times$ depth of coverage $(\sim 4.5 \mathrm{~Gb})$ on the Illumina HiSeq 2500 NGS platform in the $2 \times 100$ base read format. DNA extraction was performed by $\mathrm{Pa}-$ leo-DNA Laboratory (Lakehead University, Canada) and exome sequencing was conducted by Omega Bioservices (Norcross, GA). The cumulative exome coverage for $>25 \times$ depth of coverage was $93 \%$ indicating that almost all of the exome was accessible for probing. The variant call and annotation were performed by Genome Technology Access Center (GTAC, Washington University in St. Louis) using SnpSift varType and ANNOVAR. The resultant data were converted into the Microsoft Excel format and pathologic (deleterious) variants were identified through the five consecutive filtering steps described in [12] [13]. Functional annotation of the remaining variants was performed using UniProtKB Protein, Google Scholar, and PubMed database searches.

\section{Results}

\subsection{Anatomical Characterization}

A body of an 83-year-old individual with the male sex assigned at birth was received through the Gift of Body Program at the Center for Anatomical Science and Education (CASE) of the SLU School of Medicine, USA. The records indicated that this individual self-identified as a female by legally changing his first and middle names to female ones later in his life and his death certificate listed his sex as a female. It was also self-reported by the body donor that in the adulthood he/she underwent a complete penectomy without further specification. The 
body was hairless with an exception of a sparse hair on the scalp (Figure 1(A)), had a large scrotum with no penis observed (Figure 1(B)), and the urethral orifice was identified posterior to the scrotum (Figure 1(C)). A scrotum dissection revealed that the ductus deferens, epididymis, and testes were all intact (Figure 2(A)). However, the latter were much smaller than normal with the volume $\sim 3.3$ $\mathrm{cm}^{3}$ (Figure 2(A)) versus $\sim 7.5 \mathrm{~cm}^{3}$ for the age matched normal individuals [14]. Dissection of the pelvic cavity revealed normal male anatomy with the seminal vesicles and ductus deferens located posterior to the bladder (Figure 2(B)). The prostate was also intact, positioned inferiorly to the bladder (Figure 2(C)).

\subsection{Histological Analysis}

Histological analysis of testicular tissue revealed abnormal seminiferous tubule epithelium. As compared to the age matched control body, the epithelium was devoid of germ and Sertoli cells and Leydig cells were not observed in the interstitium (Figure 2(D)).

\subsection{Bone Densitometry}

Because the results of histological analysis were indicative of testicular malformation and, consequently, hormone deficiency with the latter being commonly linked to osteoporosis, the bone mineral density in the cadaveric body was assessed by dual-energy X-ray absorptiometry (DEXA) scanning of the lumbar spine and left femoral neck yielding the respective T-score values of -3.1 and -3.4 . These values are characteristic of osteoporosis (http://www.bones.nih.gov) and may serve as an indirect measure of the hormone deficiency in the examined body.

\subsection{Genetic Analysis}

The assessment of this individual karyotype was impossible due to incompatibility of the available methodologies with the biological material procured from the embalmed cadaveric body. However, the measured individual's height of $1.73 \mathrm{~m}$ was significantly lower as compared to that of $1.83 \mathrm{~m}$ average for the Klinefelter syndrome patients [15], which along with the absence of other phenotypic characteristics such as gynecomastia [15] (Figure 1(A)) allows, although incomplete, exclusion of the respective $47, \mathrm{XXY}$ chromosomal aberration from further consideration.

The putative genetic underlining of the present TF case was addressed by sequencing the entire coding regions of DNA (exome) extracted from the embalmed cadaveric tissue using NGS Illumina platform as described previously [12] [13]. The rare genetic variants (minor allele frequency, MAF $\leq 0.01$ ) linked to deleterious (pathological) amino acid substitutions in the mutant proteins were identified through the five sequential, stringent filtering steps described in [12] [13]. In total, the genetic screen yielded 144 deleterious (pathological) variants of 137 genes with none of them associated with either penile or urethral cancer, or the genes known to regulate the androgen hormone axis (Table S1). 

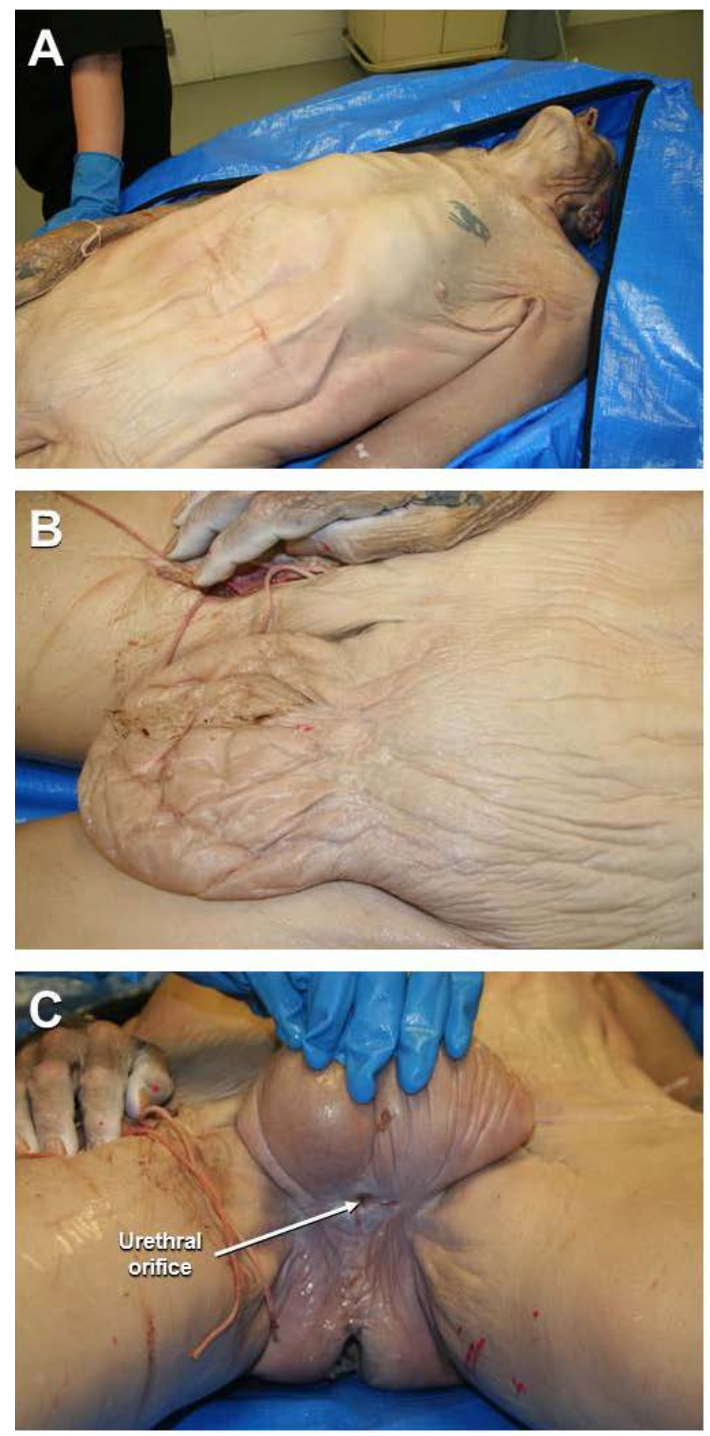

Figure 1. Physical examination of the TF body. (A): The torso view shows hairless body was with no evidence of gynecomastia. Pelvic area view demonstrates large scrotum with no penis (B) and transposed urethral orifice located posterior to the large, reflected scrotum (C).

However, six genetic variants, TPO, BBS12, DNAH9, ITF81, OFD1, and TAPT1 could be linked directly to our case (Table 1 ) due to their involvement in the regulation of a thyroid hormone production $(T P O)$ and male sex organ development through the control of cilia function (BBS12, DNAH9, ITF81, OFD1, and TAPT1) [12].

\section{Discussion}

The condition presented in the current report could be described as TGism with its progression to GD. The latter conclusion was based on the absence of genetic variants known to be associated with either penile or urethral cancer which puts forward an elective surgery as a primary cause for the reported complete penectomy. 

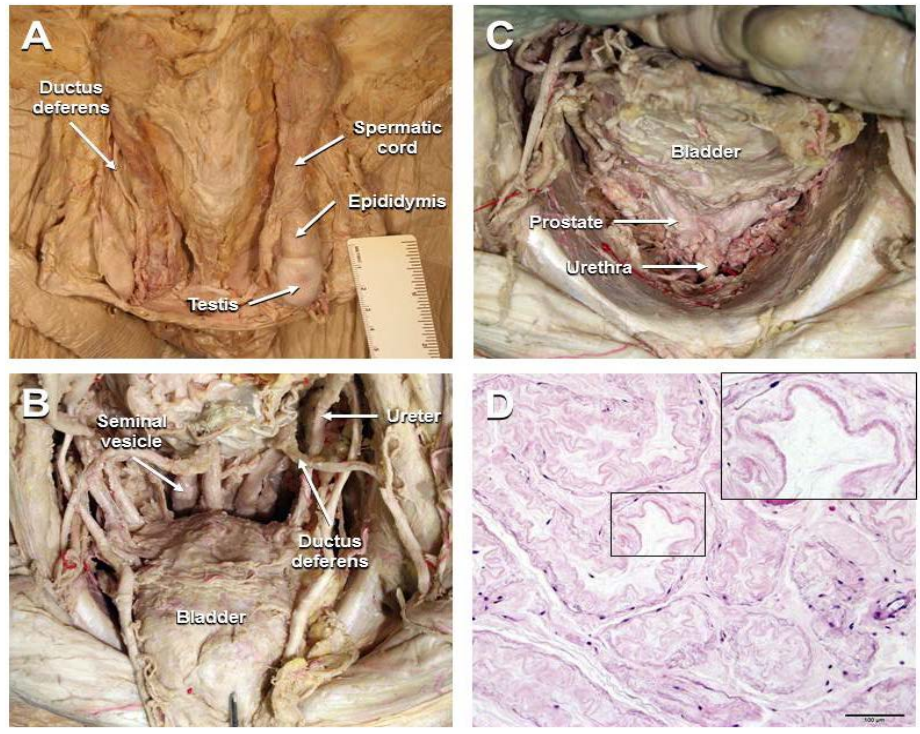

Figure 2. Gross dissection of the pelvic area and histology of the seminiferous tubules of the TF body. (A): Scrotum dissection revealed intact ductus deferens, epididymis, and small testes. Pelvic dissection revealed normal location of seminal vesicles and ductus deferens (B) as well as intact but small prostate (C). (D): Abnormal seminiferous tubule epithelium lacking germ and Sertoli cells. Leydig cells were not observed in the interstitium. Boxed area is magnified in the upper right corner.

Table 1. Selected deleterious (pathological) variants associated with the current TF.

\begin{tabular}{|c|c|c|}
\hline Gene & Protein Function & Variant \\
\hline$T P O$ & $\begin{array}{l}\text { Thyroid peroxidase. Iodination and coupling of the } \\
\text { hormonogenic tyrosines in thyroglobulin to yield the } \\
\text { thyroid hormones } \mathrm{T} 3 \text { and } \mathrm{T} 4 \text {. }\end{array}$ & $\begin{array}{c}\text { c.1181_1182insCGGC: } \\
\text { p.D394fs }\end{array}$ \\
\hline$B B S 12$ & $\begin{array}{l}\text { Bardet-Biedl syndrome } 12 \text { protein. Component of the } \\
\text { chaperonin-containing T-complex (TRiC), a molecular } \\
\text { chaperone complex that assists the folding of proteins upon } \\
\text { ATP hydrolysis. As part of the TRiC complex may play a } \\
\text { role in the assembly of BBSome, a complex involved in } \\
\text { ciliogenesis regulating transports vesicles to the cilia. }\end{array}$ & c.T116C:p.I39T \\
\hline DNAH9 & $\begin{array}{l}\text { Dynein heavy chain } 9 \text {, axonemal. Force generating protein } \\
\text { of respiratory cilia. Produces force towards the minus ends } \\
\text { of microtubules. }\end{array}$ & c.T9534G:p.F3178L \\
\hline ITF81 & $\begin{array}{l}\text { Intraflagellar transport protein } 81 \text { homolog. Component of } \\
\text { the intraflagellar transport (IFT) complex B: together with } \\
\text { IFT74, forms a tubulin-binding module that specifically } \\
\text { mediates transport of tubulin within the cilium. }\end{array}$ & c.174delA:p.58fs \\
\hline$O F D 1$ & $\begin{array}{l}\text { Oral-facial-digital syndrome } 1 \text { protein. Component of the } \\
\text { centrioles controlling mother and daughter centrioles } \\
\text { length. Involved in the biogenesis of the cilium, a } \\
\text { centriole-associated function. }\end{array}$ & c.702delA:p.A234fs \\
\hline$T A P T 1$ & $\begin{array}{l}\text { Transmembrane anterior posterior transformation protein } \\
1 \text { homolog. Plays a role in primary cilia formation. }\end{array}$ & c.A704C:p.H235P \\
\hline
\end{tabular}


It is hypothesized herein that one of the important underlinings of the above condition could be linked to the impaired T3 and T4 thyroid hormone production which could negatively affect a cross-talk between the thyroid and androgen hormone axes [16] [17] thereby potentially leading to anatomical and physiological alterations similar to those in PAIS [9]. This hypothesis is based on: 1) a presence of TPO deleterious (pathological) variant that control the T3 and T4 production and the absence of the variants that regulate the androgen hormone axis (Table 1$) ; 2$ ) the observed testicular malformation at both the anatomical and histological levels (Figure 2); and 3) the T-score values derived for the lumbar spine $(-3.1)$ and femoral neck (-3.4) being comparable to those of patients with PAIS [18] while being much worse than those of older male subjects with low testosterone levels, respectively -0.48 and -1.56 [19]. Importantly, the T3 and $\mathrm{T} 4$ thyroid deficiency has been recently reported in a patient with GD and normal, 46, XX, female karyotype [20]. With this regard, the data presented in the current report raise a very important question is to whether the thyroid hormone deficiency could be viewed as one of the important biological underlinings of TGism and GD. It should also be noted, that prenatal maternal thyroid deficiency has been previously linked to impacting sexuality [21] [22].

The detected deleterious (pathological) BBS12, DNAH9, ITF81, OFD1, and $T A P T 1$ variants known to negatively affect cilia development and function could serve as a prerequisite for other male sex organ malformations ambiguously present in the current case, such as hypospadias (Figure 1(B)) [12] and complete penoscrotal transposition [23]. Intriguingly, in the model organisms, cilium has also been shown to be a major regulator of male sexual behavior such as mate searching and selection [24] [25] [26].

\section{Conclusion}

The results reported in the current study provide unique information for the hypothesis that thyroid hormone deficiency could be one of the important biological underlinings of TGism and GD that merits its further evaluation in a clinical setting.

\section{Acknowledgements}

We gratefully acknowledge Dr. Paul Cliften (GTAC, Washington University in St. Louis, St. Louis, MO, USA) for his invaluable assistance with the bioinformatics analysis as well as Caroline Murphy and Barbara Nagel (SLU) for their skillful help with the histology slides preparation. Dr. Maria Teresa Tersigni-Tarrant is acknowledged for her assistance with the anatomical dissection.

\section{Limitations}

The deleterious (pathologic) genetic variants were identified by the exome sequencing of a single proband. The high scientific value of a single proband approach to unique human cases has been recently demonstrated [27]. 


\section{Authors' Contributions}

AF designed the study, coordinated genetics data collection and bioinformatics analysis, analyzed the data, wrote and revised the manuscript. LP participated in designing the study, collected and analyzed the anatomical and bone densitometry data, and performed functional annotation of genetic variants. CL analyzed the histological data. YT assisted with the anatomical data collection and analysis. JRM designed and supervised the study, analyzed the data, wrote and revised the manuscript.

\section{Funding}

This study was supported by the Center for Anatomical Science and Education, SLU School of Medicine.

\section{Disclosure}

These data were presented in part at the Annual Experimental Biology Meeting (FASEB J. (2019), 33: Suppl. 1, Abstract lb109).

\section{Conflicts of Interest}

The authors declare no conflicts of interest regarding the publication of this paper.

\section{References}

[1] T'Sjoen, G., et al. (2019) Endocrinology of Transgender Medicine. Endocrine Reviews, 40, 97-117. https://doi.org/10.1210/er.2018-00011

[2] Flores, A.R., et al. (2016) How Many Adults Identify as Transgender in the United States. The Williams Institute, Los Angelos, 1-13.

[3] Fernandez, R., et al. (2018) Molecular Basis of Gender Dysphoria: Androgen and Estrogen Receptor Interaction. Psychoneuroendocrinology, 98, 161-167. https://doi.org/10.1016/j.psyneuen.2018.07.032

[4] Yang, F., et al. (2017) Genomic Characteristics of Gender Dysphoria Patients and Identification of Rare Mutations in RYR3 Gene. Scientific Reports, 7, Article No. 8339. https://doi.org/10.1038/s41598-017-08655-X

[5] Bentz, E.K., et al. (2008) A Polymorphism of the CYP17 Gene Related to Sex Steroid Metabolism Is Associated with Female-to-Male But Not Male-to-Female Transsexualism. Fertil Steril, 90, 56-59. https://doi.org/10.1016/j.fertnstert.2007.05.056

[6] Fernandez, R., et al. (2018) Analyses of Karyotype by G-Banding and High-Resolution Microarrays in a Gender Dysphoria Population. Genes Genomics, 40, 465-473. https://doi.org/10.1007/s13258-017-0646-0

[7] Diamond, D.A. and Yu, R.N. (2016) Disorders of Sexual Development: Etiology, Evaluation, and Medical Management. In: Wein, A.J., Kavoussi, L.R., Partin, A.W. and Peters, C.A., Eds., Campbell- Walsh Urology, Elsevier, Philadelphia, 3469-3497.

[8] O’Hanlan, K.A., Gordon, J.C. and Sullivan, M.W. (2018) Biological Origins of Sexual Orientation and Gender Identity: Impact on Health. Gynecologic Oncology, 149, 33-42. https://doi.org/10.1016/j.ygyno.2017.11.014 
[9] Achermann, J.C. and Hughes, I.A. (2016) Pediatric Disorders of Sex Development. In: Melmed, S., et al., Eds., Williams Textbook of Endocrinology, Elsevier, Philadelphia, 893-963.

[10] Foreman, M., et al. (2019) Genetic Link between Gender Dysphoria and Sex Hormone Signaling. The Journal of Clinical Endocrinology \& Metabolism, 104, 390-396. https://doi.org/10.1210/jc.2018-01105

[11] Detton, A.J. (2017) Grant's Dissector. 16th Edition, Walters Kluwer, Alphen aan den Rijn.

[12] Frolov, A., Tan, Y., Rana, M. and Martin, J.R. (2018) A Rare Case of Human Diphallia Associated with Hypospadias. Case Reports in Urology, 2018, Article ID: 8293036. https://doi.org/10.1155/2018/8293036

[13] Jenkins, M., et al. (2019) Situs Inversus Totalis in a 96-Year-Old Female Cadaver: Evidence Pointing toward the Two-Cilia Model. Italian Journal of Anatomy and Embryology, 124, 230-246.

[14] Yang, H., et al. (2011) The Effects of Aging on Testicular Volume and Glucose Metabolism: An Investigation with Ultrasonography and FDG-PET. Molecular Imaging and Biology, 13, 391-398. https://doi.org/10.1007/s11307-010-0341-x

[15] Kamischke, A., et al. (2003) Clinical and Diagnostic Features of Patients with Suspected Klinefelter Syndrome. Journal of Andrology, 24, 41-48.

[16] Donnelly, P. and White, C. (2000) Testicular Dysfunction in Men with Primary Hypothyroidism; Reversal of Hypogonadotrophic Hypogonadism with Replacement Thyroxine. Clinical Endocrinology, 52, 197-201. https://doi.org/10.1046/j.1365-2265.2000.00918.x

[17] Flood, D.E., Fernandino, J.I. and Langlois, V.S. (2013) Thyroid Hormones in Male Reproductive Development: Evidence for Direct Crosstalk between the Androgen and Thyroid Hormone Axes. General and Comparative Endocrinology, 192, 2-14. https://doi.org/10.1016/j.ygcen.2013.02.038

[18] Soule, S.G., et al. (1995) Osteopenia as a Feature of the Androgen Insensitivity Syndrome. Clinical Endocrinology, 43, 671-675. https://doi.org/10.1111/j.1365-2265.1995.tb00533.x

[19] Amory, J.K., et al. (2004) Exogenous Testosterone or Testosterone with Finasteride Increases Bone Mineral Density in Older Men with Low Serum Testosterone. The Journal of Clinical Endocrinology \& Metabolism, 89, 503-510. https://doi.org/10.1210/jc.2003-031110

[20] Beatrice, A., Sumana, K. and Mengade, M.G. (2019) Juvenile Autoimmune Hypothyroidism and Gender Dysphoria: A Causal Relationship or a Casual Coincidence? The Indian Journal of Endocrinology and Metabolism, 23, 166-167. https://doi.org/10.4103/ijem.IJEM_656_18

[21] Sabuncuoglu, O. (2015) High Rates of Same-Sex Attraction/Gender Nonconformity in the Offspring of Mothers with Thyroid Dysfunction during Pregnancy: Proposal of Prenatal Thyroid Model. Mental Illness, 7, 5810. https://doi.org/10.4081/mi.2015.5810

[22] Sabuncuoglu, O. (2017) Towards a Further Understanding of Prenatal Thyroid Theory of Homosexuality: Autoimmune Thyroiditis, Polycystic Ovary Syndrome, Autism and Low Birth Weight. Mental Illness, 9, 7325. https://doi.org/10.4081/mi.2017.7325

[23] Sexton, P., et al. (2015) Complete Penoscrotal Transposition: Case Report and Review of the Literature. Fetal Diagnosis and Therapy, 37, 70-74.

https://doi.org/10.1159/000358592 
[24] Hilbert, Z.A. and Kim, D.H. (2017) Sexually Dimorphic Control of Gene Expression in Sensory Neurons Regulates Decision-Making Behavior in C. elegans. Elife, 6, e21166. https://doi.org/10.7554/eLife.21166

[25] Peden, E.M. and Barr, M.M. (2005) The KLP-6 Kinesin Is Required for Male Mating Behaviors and Polycystin Localization in Caenorhabditis elegans. Current Biology, 15, 394-404. https://doi.org/10.1016/j.cub.2004.12.073

[26] White, J.Q., et al. (2007) The Sensory Circuitry for Sexual Attraction in C. elegans Males. Current Biology, 17, 1847-1857. https://doi.org/10.1016/j.cub.2007.09.011

[27] Arboleda-Velasquez, J.F., et al. (2019) Resistance to Autosomal Dominant Alzheimer's Disease in an APOE3 Christchurch Homozygote: A Case Report. Nature Medicine, 25, 1680-1683. https://doi.org/10.1038/s41591-019-0611-3 


\section{Supplementary Materials}

The file contains Table S1 (Complete list of deleterious (pathologic) genetic variants associated with the current TF).

Table S1. Complete list of deleterious (pathologic) genetic variants associated with the current TF.

\begin{tabular}{cl}
\hline Gene & \multicolumn{1}{c}{ Protein Function } \\
\hline \multirow{3}{*}{$A B C A 13$} & $\begin{array}{l}\text { ATP-binding cassette sub-family A member 13. ATPase activity, coupled to } \\
\text { transmembrane movement of substances. Lipid transport. Neutrophil } \\
\text { degranulation. }\end{array}$ \\
ABCC1 & $\begin{array}{l}\text { Multidrug resistance-associated protein 1. Mediates export of organic anions and } \\
\text { drugs from the cytoplasm. Hydrolyzes ATP with low efficiency. }\end{array}$ \\
& $\begin{array}{l}\text { Tyrosine-protein kinase ABL1. Non-receptor tyrosine-protein kinase that plays a } \\
\text { role in many key processes linked to cell growth and survival such as cytoskeleton } \\
\text { remodeling in response to extracellular stimuli, cell motility and adhesion, receptor } \\
\text { endocytosis, autophagy, DNA damage response and apoptosis. }\end{array}$
\end{tabular}
endocytosis, autophagy, DNA damage response and apoptosis.

A disintegrin and metalloproteinase with thrombospondin motifs 9. Cleaves the large aggregating proteoglycans, aggrecan and versican.

Has a protease-independent function in promoting the transport from the endoplasmic reticulum to the Golgi apparatus of a variety of secretory cargos.

Adipogenin. Brown fat cell differentiation. Positive regulation of fat cell differentiation. Spermatogenesis.

Cytosolic carboxypeptidase 4. Metallocarboxypeptidase that mediates deglutamylation of target proteins. Catalyzes the deglutamylation of polyglutamate side chains generated by post-translational polyglutamylation in proteins such as tubulins.

ANKRD20A1 Ankyrin repeat domain-containing protein 20A1.

$A N K R D 20 A 3$ Ankyrin repeat domain-containing protein 20A3.

Rho GTPase-activating protein 21. Functions as a GTPase-activating protein

ARHGAP21 (GAP) for RHOA and CDC42. Downstream partner of ARF1 which may control Golgi apparatus structure and function

$A R S D \quad$ Arylsulfatase D. Glycosphingolipid metabolism.

$B A G E 2 \quad B$ melanoma antigen 2. Candidate gene encoding tumor antigens.

$B A G E 3 \quad$ B melanoma antigen 3. Candidate gene encoding tumor antigens.

Bardet-Biedl syndrome 12 protein. Component of the chaperonin-containing T-complex (TRiC), a molecular chaperone complex that assists the folding of

BBS12 proteins upon ATP hydrolysis. As part of the TRiC complex may play a role in the assembly of BBSome, a complex involved in ciliogenesis regulating transports vesicles to the cilia (PubMed: 20080638).

$B C L A F 1 \quad$ Bcl-2-associated transcription factor 1. Death-promoting transcriptional repressor.

BZRAP1 BZRAP1 protein. Benzodiazepine receptor binding.

Complement C4-A. Non-enzymatic component of C3 and C5 convertases and thus essential for the propagation of the classical complement pathway. Covalently binds to immunoglobulins and immune complexes and enhances the solubilization of immune aggregates and the clearance of IC through CR1 on erythrocytes. 


\section{Continued}

$C A B$

$C 4 B \_2$

$C A C N A 2 D$

$C B L C$

CD200R1L

$C D C 27$

CEPT1

$C G N$

$C N G A 2$

CNTRL

COL11A1

$C Y P 1 A 2$
Complement C4-B.

Complement C4-B2.

Complement component C9. Constituent of the membrane attack complex (MAC) that plays a key role in the innate and adaptive immune response by forming pores in the plasma membrane of target cells (PubMed:9634479, PubMed:9212048, PubMed: 26841934).

Voltage-dependent calcium channel subunit alpha-2/delta-3. The alpha-2/delta subunit of voltage-dependent calcium channels regulates calcium current density and activation/inactivation kinetics of the calcium channel. Acts as a regulatory subunit for P/Q-type calcium channel (CACNA1A),

E3 ubiquitin-protein ligase CBL-C. Acts as an E3 ubiquitin-protein ligase, which accepts ubiquitin from specific E2 ubiquitin-conjugating enzymes, and then transfers it to substrates promoting their degradation by the proteasome. Functionally coupled with the E2 ubiquitin-protein ligases UB2D1, UB2D2 and UB2D3. Regulator of EGFR mediated signal transduction; upon EGF activation, ubiquitinates EGFR. Isoform 1, but not isoform 2, inhibits EGF stimulated MAPK1 activation.

$C C D C 144 N L$ Putative coiled-coil domain-containing protein $144 \mathrm{~N}$-terminal-like. Could be the $C C D C 144 N L$ product of a pseudogene. Shares high sequence similarity with the N-terminus of $C C D C 144 N L$ the CCDC144 family.

Serine-rich coiled-coil domain-containing protein 2. Microtubule-binding protein which might play a role in microtubule bundling.

Cell surface glycoprotein CD200 receptor 2. Regulates neuroinflammatory response.

Cell division cycle protein 27 homolog. Component of the anaphase promoting complex/cyclosome (APC/C), a cell cycle-regulated E3 ubiquitin ligase that controls progression through mitosis and the $\mathrm{G} 1$ phase of the cell cycle.

Choline/ethanolaminephosphotransferase 1. Catalyzes both phosphatidylcholine and phosphatidylethanolamine biosynthesis from CDP-choline and CDP-ethanolamine, respectively. Involved in protein-dependent process of phospholipid transport to distribute phosphatidyl choline to the lumenal surface.

Cingulin. Probably plays a role in the formation and regulation of the tight junction (TJ) paracellular permeability barrier.

Cyclic nucleotide-gated olfactory channel. Odorant signal transduction is probably mediated by a G-protein coupled cascade using cAMP as second messenger.

Centriolin. Involved in cell cycle progression and cytokinesis. During the late steps of cytokinesis, anchors exocyst and SNARE complexes at the midbody, thereby allowing secretory vesicle-mediated abscission.

Collagen alpha-1(XI) chain. May play an important role in fibrillogenesis by controlling lateral growth of collagen II fibrils.

Cytochrome P450 1A2. Cytochromes P450 are a group of heme-thiolate monooxygenases. In liver microsomes, this enzyme is involved in an NADPH-dependent electron transport pathway. It oxidizes a variety of structurally unrelated compounds, including steroids, fatty acids, and xenobiotics. 


\section{Continued}

DNAH9 Produces force towards the minus ends of microtubules. Dynein has ATPase activity; the force-producing power stroke is thought to occur on release of ADP.

DPH6

EFTUD1

FAM104A

FAM63A

FAM81B

FOXD4L6

FPR2

$G A G E 13$

GAGE2E

GAGE8

$G G T 1$

$G G T 1$

$G G T 1$

GRHL3

GTF3C

$G Y G 1$

$H L A-A$

IFT81

$\mathrm{JPH} 4$
Diphthine--ammonia ligase. Amidase that catalyzes the last step of diphthamide biosynthesis using ammonium and ATP.

Elongation factor-like GTPase 1. Involved in the biogenesis of the 60S ribosomal subunit and translational activation of ribosomes.

Protein FAM104A. Highest level of expression is in sperm.

Protein FAM63A, aka MINDY1. Ubiquitin carboxyl-terminal hydrolase MINDY-1. Hydrolase that can specifically remove "Lys-48"-linked conjugated ubiquitin from proteins. May play a regulatory role at the level of protein turnover.

\section{Protein FAM81B}

Forkhead box protein D4-like 6. Transcription factor. Anatomical structure morphogenesis. Cell differentiation.

Formyl peptide receptor 2 . High affinity receptor for $\mathrm{N}$-formyl-methionyl peptides (FMLP), which are powerful neutrophil chemotactic factors (PubMed: 12218158, PubMed: 10477558, PubMed: 19387439). Receptor for the chemokine-like protein FAM19A5, mediating FAM19A5-stimulated macrophage chemotaxis and the inhibitory effect on TNFSF11/RANKL-induced osteoclast differentiation (PubMed: 29138422).

$\mathrm{G}$ antigen 13. This gene belongs to a multigene family expressed in a large variety of tumors whereas in normal tissues, expression is restricted to germ cells.

G antigen $2 \mathrm{E}$

G antigen 8 .

Glutathione hydrolase 1 proenzyme. Initiates extracellular glutathione (GSH) breakdown, provides cells with a local cysteine supply and contributes to maintain intracellular GSH level. It is part of the cell antioxidant defense mechanism.

Grainyhead-like protein 3 homolog. Transcription factor playing important roles in primary neurulation and in the differentiation of stratified epithelia of both ectodermal and endodermal origin.

General transcription factor 3C polypeptide 1. Required for RNA polymerase III-mediated transcription.

Glycogenin-1. Self-glucosylates, via an inter-subunit mechanism, to form an oligosaccharide primer that serves as substrate for glycogen synthase.

MHC class I HLA-A protein. Involved in the presentation of foreign antigens to the immune system.

Intraflagellar transport protein 81 homolog. Component of the intraflagellar transport (IFT) complex B: together with IFT74, forms a tubulin-binding module that specifically mediates transport of tubulin within the cilium. Required for ciliogenesis (PubMed: 27666822, PubMed: 23990561). Required for proper regulation of SHH signaling (PubMed: 27666822).

Junctophilin-4. Junctophilins contribute to the formation of junctional membrane complexes (JMCs) which link the plasma membrane with the endoplasmic or sarcoplasmic reticulum in excitable cells. Provides a structural foundation for functional cross-talk between the cell surface and intracellular calcium release channels. 


\section{Continued}

\begin{tabular}{cl}
\hline \multirow{2}{*}{ KCNG4 } & $\begin{array}{l}\text { Potassium voltage-gated channel subfamily G member 4. Potassium channel } \\
\text { subunit that does not form functional channels by itself. Can form functional } \\
\text { heterotetrameric channels with KCNB1. Regulates ion transmembrane transport. }\end{array}$ \\
KIAA1549L & UPF0606 protein KIAA1549L.
\end{tabular}

$K I R 2 D L 1$

KIR2DL1

KIR2DL3

KIR2DS4

KIR2DS4

KIR2DS4

KRTAP10-6

LCN12

LDLRAD4

LIPC

LPIN3

MAML3

MRGPRX1

Mas-related G-protein coupled receptor member X1. Orphan receptor activated by neuropeptides terminating in Arg-Phe or Arg-Phe-amideMay regulate the function of nociceptive neurons by modulation of pain perception.

$M S T 1 L$

$M S T 1 L$

$M U C 4$

$M U C A$

MYO1H

$N B P F 10$

NBPF12

$N B P F 12$

NBPF 20

Killer cell immunoglobulin-like receptor 2DL1. Receptor on natural killer (NK) cells for some HLA-C alleles such as w4 and w6. Inhibits the activity of NK cells thus preventing cell lysis.

Killer cell immunoglobulin-like receptor 2DL3. Receptor on natural killer (NK) cells for HLA-C alleles (HLA-Cw1, HLA-Cw3 and HLA-Cw7). Inhibits the activity of NK cells thus preventing cell lysis.

Killer cell immunoglobulin-like receptor 2DS4. Receptor on natural killer (NK) cells for HLA-C alleles. Does not inhibit the activity of NK cells.

Keratin-associated protein 10-6. In the hair cortex, hair keratin intermediate filaments are embedded in an interfilamentous matrix, consisting of hair keratin-associated proteins (KRTAP), which are essential for the formation of a rigid and resistant hair shaft through their extensive disulfide bond cross-linking with abundant cysteine residues of hair keratins.

Epididymal-specific lipocalin-12. Binds all-trans retinoic acid and may act as a retinoid carrier protein within the epididymis. May play a role in male fertility (By similarity).

Low-density lipoprotein receptor class A domain-containing protein 4. Functions as a negative regulator of TGF-beta signaling and thereby probably plays a role in cell proliferation, differentiation, apoptosis, motility, extracellular matrix production and immunosuppression.

Hepatic triacylglycerol lipase. Hepatic lipase has the capacity to catalyze hydrolysis of phospholipids, mono-, di-, and triglycerides, and acyl-CoA thioesters. It is an important enzyme in HDL metabolism.

Phosphatidate phosphatase LPIN3. Regulates fatty acid metabolism. Magnesium-dependent phosphatidate phosphatase enzyme which catalyzes the conversion of phosphatidic acid to diacylglycerol during triglyceride, phosphatidylcholine and phosphatidylethanolamine biosynthesis (By similarity).

Mastermind-like protein 3. Acts as a transcriptional coactivator for NOTCH proteins. Has been shown to amplify NOTCH-induced transcription of HES1.

Putative macrophage stimulating 1-like protein.

Mucin-4. May play a role in tumor progression. Ability to promote tumor growth may be mainly due to repression of apoptosis as opposed to proliferation. Has anti-adhesive properties.

Unconventional myosin-Ih. Represents an unconventional myosin.

Neuroblastoma breakpoint family member 10.. RNA binding.

Neuroblastoma breakpoint family member 12 . RNA binding.

Neuroblastoma breakpoint family member 20. RNA binding. 


\section{Continued} b558 are required for activation of the latent NADPH oxidase (necessary for superoxide production).

Nebulette. Binds to actin and plays an important role in the assembly of the Z-disk. NEBL May functionally link sarcomeric actin to the desmin intermediate filaments in the heart muscle sarcomeres (PubMed: 27733623).

NFATC1 Isoform 2 might play a role in the assembly of focal adhesion (PubMed: 15004028).

Neuroplastin. Probable homophilic and heterophilic cell adhesion molecule involved in long term potentiation at hippocampal excitatory synapses through activation of p38MAPK. May also regulate neurite outgrowth by activating the FGFR1 signaling pathway. May play a role in synaptic plasticity (By similarity).

Uridine diphosphate glucose pyrophosphatase. Hydrolyzes UDP-glucose to glucose

NUDT22 1-phosphate and UMP and UDP-galactose to galactose 1-phosphate and UMP. Preferred substrate is UDP-glucose.

Nuclear pore complex protein Nup153. Component of the nuclear pore complex (NPC), a complex required for the trafficking across the nuclear envelope.

Nucleoporin p58/p45. Component of the nuclear pore complex, a complex required for the trafficking across the nuclear membrane.

Oral-facial-digital syndrome 1 protein. Component of the centrioles controlling mother and daughter centrioles length. Recruits to the centriole IFT88 and centriole distal appendage-specific proteins including CEP164. Involved in the biogenesis of the cilium, a centriole-associated function. Plays an important role in development by regulating Wnt signaling and the specification of the left-right axis.

OPALIN Transmembrane protein.

OR11H1 Olfactory receptor $11 \mathrm{H} 1$

$O R 6 N 2 \quad$ Olfactory receptor $6 \mathrm{~N} 2$

Protocadherin alpha-8. Potential calcium-dependent cell-adhesion protein. May be involved in the establishment and maintenance of specific neuronal connections in the brain.

Phosphoenolpyruvate carboxykinase [GTP], mitochondrial. Catalyzes the conversion of oxaloacetate (OAA) to phosphoenolpyruvate (PEP), the rate-limiting step in the metabolic pathway that produces glucose from lactate and other precursors derived from the citric acid cycle.

Phosducin-like protein 3. Acts as a chaperone for the angiogenic VEGF receptor

PDCL3 KDR/VEGFR2, controlling its abundance and inhibiting its ubiquitination and degradation. Modulates the activation of caspases during apoptosis.

Peroxisome assembly factor 2. Involved in peroxisome biosynthesis.

PGM2L1 Glucose 1,6-bisphosphate synthase.

PLA2G4C

Cytosolic phospholipase A2 gamma. Has a preference for arachidonic acid at the sn-2 position of phosphatidylcholine as compared with palmitic acid.

1-phosphatidylinositol 4,5-bisphosphate phosphodiesterase delta-4. Hydrolyzes the molecules diacylglycerol (DAG) and inositol 1,4,5-trisphosphate (IP3). 


\section{Continued}

PLXNB3

Plexin-B3. Receptor for SEMA5A that plays a role in axon guidance, invasive growth and cell migration.

POTEC POTE ankyrin domain family member C. Highest expression level is in testis.

PPIAL4G

Peptidyl-prolyl cis-trans isomerase A-like 4G. PPIases accelerate the folding of proteins.

PRAMEF18

PRAME family member 18 . Negative apoptosis regulation. Negative regulation of cell differentiation.

PRAMEF22

PRAME family member 22. Negative apoptosis regulation. Negative regulation of cell differentiation.

Perforin-1. Plays a key role in secretory granule-dependent cell death, and in defense against virus-infected or neoplastic cells. Plays an important role in killing other cells that are recognized as non-self by the immune system, e.g. in transplant rejection or some forms of autoimmune disease.

Proteasome subunit alpha-type 8. Component of the spermatoproteasome, a form of the proteasome specifically found in testis that promotes degradation of histones, thereby participating actively to the exchange of histones during spermatogenesis.

$R B M X$

RNA-binding motif protein, $\mathrm{X}$ chromosome. RNA-binding protein that plays several role in the regulation of pre- and post-transcriptional processes.

$R B M X L 1$

RNA binding motif protein, X-linked-like-1. RNA-binding protein which may be involved in pre-mRNA splicing.

Regulator of G-protein signaling 11. Inhibits signal transduction by increasing the $R G S 11$ GTPase activity of $\mathrm{G}$ protein alpha subunits thereby driving them into their inactive GDP-bound form.

RIT2

GTP-binding protein Rit2. Binds and exchanges GTP and GDP. Binds and modulates the activation of POU4F1 as gene expression regulator.

$R L N 3$

Relaxin-3. May play a role in neuropeptide signaling processes. Ligand for LGR7, relaxin-3 receptor-1 (GPCR135) and relaxin-3 receptor-2 (GPCR142).

SAMD10 Sterile alpha motif domain-containing protein 10.

SCN9A

Sodium channel protein type 9 subunit alpha. Plays a role in pain mechanisms, especially in the development of inflammatory pain.

Probable helicase senataxin. Probable RNA/DNA helicase involved in diverse aspects of RNA metabolism and genomic integrity. Plays a role in neurite outgrowth in hippocampal cells through FGF8-activated signaling pathways. Inhibits retinoic acid-induced apoptosis (PubMed: 21576111).

$S F X N 1$

Sideroflexin-1. Might be involved in the transport of a component required for iron utilization into or out of the mitochondria.

Solute carrier family 22 member 13 . Nicotinate transmembrane transporter activity.

SLC23A2 Solute carrier family 23 member 2. L-ascorbate:sodium symporter activity.

SLC25A5 ADP/ATP translocase 2. Catalyzes the exchange of cytoplasmic ADP with

$S L C 25 A 5$ mitochondrial ATP across the mitochondrial inner membrane. 


\section{Continued}

\begin{tabular}{|c|c|}
\hline$S L C 9 B 1$ & $\begin{array}{l}\text { Sodium/hydrogen exchanger 9B1. Sperm-specific } \mathrm{Na}+\mathrm{H}+\text { exchanger involved in } \\
\text { intracellular pH regulation of spermatozoa. Involved in sperm motility and } \\
\text { fertility. }\end{array}$ \\
\hline$S L F N 13$ & $\begin{array}{l}\text { Schlafen family member 13. Endoribonuclease that cleaves tRNAs and rRNAs } \\
\text { (PubMed:29563550). }\end{array}$ \\
\hline$S O R D$ & Sorbitol dehydrogenase. Oxidoreductase activity. Zinc ion binding. \\
\hline$S P A T A 31 A 1$ & Spermatogenesis-associated protein $31 \mathrm{~A} 1$. May play a role in spermatogenesis. \\
\hline SPATA31C1 & $\begin{array}{l}\text { Putative spermatogenesis-associated protein } 31 \mathrm{Cl} \text {. May play a role in } \\
\text { spermatogenesis. }\end{array}$ \\
\hline$S P A T A 31 C 2$ & $\begin{array}{l}\text { Putative spermatogenesis-associated protein } 31 \mathrm{C} 2 \text {. May play a role in } \\
\text { spermatogenesis. }\end{array}$ \\
\hline$S P A T S 1$ & $\begin{array}{l}\text { Spermatogenesis-associated serine-rich protein } 1 \text {. Highest expression level } \\
\text { is in testis. }\end{array}$ \\
\hline$S U G C T$ & $\begin{array}{l}\text { Succinate--hydroxymethylglutarate CoA-transferase. Catalyzes the } \\
\text { succinyl-CoA-dependent conversion of glutarate to glutaryl-CoA. }\end{array}$ \\
\hline$S Y N E 3$ & $\begin{array}{l}\text { Nesprin-3. As a component of the LINC (LInker of Nucleoskeleton and } \\
\text { Cytoskeleton) complex involved in the connection between the nuclear } \\
\text { lamina and the cytoskeleton. }\end{array}$ \\
\hline$T A P T 1$ & $\begin{array}{l}\text { Transmembrane anterior posterior transformation protein } 1 \text { homolog. } \\
\text { Plays a role in primary cilia formation (PubMed: 26365339). }\end{array}$ \\
\hline$T E X 29$ & Testis-expressed protein 29. Highest expression level is in right testis. \\
\hline THBS2 & $\begin{array}{l}\text { Thrombospondin-2. Adhesive glycoprotein that mediates cell-to-cell and } \\
\text { cell-to-matrix interactions. Ligand for CD } 36 \text { mediating antiangiogenic properties. }\end{array}$ \\
\hline$T L E 2$ & Transducin-like enhancer protein 2. Regulation of transcription. \\
\hline TM4SF19 & Transmembrane 4 L6 family member 19 . Highest expression level is in ectocervix. \\
\hline$T N X B$ & $\begin{array}{l}\text { Tenascin-X. Appears to mediate interactions between cells and the extracellular } \\
\text { matrix. Substrate-adhesion molecule that appears to inhibit cell migration. } \\
\text { Accelerates collagen fibril formation. May play a role in supporting the } \\
\text { growth of epithelial tumors. }\end{array}$ \\
\hline$T P O$ & $\begin{array}{l}\text { Thyroid peroxidase. Iodination and coupling of the hormonogenic tyrosines in } \\
\text { thyroglobulin to yield the thyroid hormones } \mathrm{T} 3 \text { and } \mathrm{T} 4 \text {. }\end{array}$ \\
\hline TUBGCP6 & $\begin{array}{l}\text { Gamma-tubulin complex component } 6 \text {. Gamma-tubulin complex is necessary for } \\
\text { microtubule nucleation at the centrosome. }\end{array}$ \\
\hline TYRO3 & $\begin{array}{l}\text { Tyrosine-protein kinase receptor TYRO3. Regulates many physiological processes } \\
\text { including cell survival, migration and differentiation. }\end{array}$ \\
\hline WHSC1 & $\begin{array}{l}\text { Histone-lysine N-methyltransferase NSD2. Histone methyltransferase with histone } \\
\text { H3 "Lys-27" (H3K27me) methyltransferase activity. Isoform } 2 \text { may act as a } \\
\text { transcription regulator that binds DNA and suppresses IL5 transcription } \\
\text { through HDAC recruitment. }\end{array}$ \\
\hline ZNF563 & Transcription regulation. \\
\hline ZNF585B & Transcription regulation. \\
\hline
\end{tabular}




\section{Continued}

ZNF717

ZNF717

ZNF717

ZNF717

ZNF717

ZNF846 Transcription regulation.

ZXDA Cooperates with CIITA to promote transcription of MHC class I and MHC class II $Z X D A$ genes. 